\title{
Zur Entstehung der Elementa Physiologiae Albrecht Hallers (1708-1777) ${ }^{1}$
}

\author{
Von Heinrich Buess
}

Nach dem Vorgange eines Ausländers, Tissot, fingen nunmehr auch die Ärzte mit Eifer an, auf die allgemeine Bildung zu wirken. Sehr großen Einfluß hatten Haller, Unzer, Zimmermann, und was man im einzelnen gegen sie, besonders gegen den letzten, auch sagen mag, sie waren zu ihrer Zeit sehr wirksam. Und davon sollte in der Geschichte, vorzüglich aber in der Biographie die Rede sein: denn nicht insofern der Mensch etwas zurückläßt, sondern insofern er wirkt und genießt und andere zu wirken und zu genießen anregt, bleibt er von Bedeutung.

Goethe, Dichtung und Wahrheit, 2. Teil, 7. Buch

Während eine frühere Studie sich zum Ziel setzte, einen Teilaspekt des ersten Handbuches der Physiologie, nämlich den krankhaft gestörten Blutkreislauf, auch in seiner Vorgeschichte kennenzulernen, möchte ich im vorliegenden Beitrag die Person des Verfassers dieses Werkes, den Berner Arzt Albreght Haller, in den Vordergrund rücken und vielleicht dadurch einen kleinen Anstoß geben zu der endlich fälligen Gesamtbiographie Hallers, einem Werk, dem nur durch Zusammenarbeit verschiedener «Fakultäten» ein Erfolg beschieden sein kann. In erster Linie ist dazu erforderlich eine umfassende Einarbeitung in den Briefwechsel Hallers mit seinen Zeitgenossen.

In diesem knappen Rahmen kann nur eben auf einige wertvolle quellenkundliche Vorarbeiten hingewiesen werden. Bekanntlich hat ja Haller selbst den Grundstein gelegt, indem er von den 13202 Briefen, die von 1209 Korrespondenten an ihn gerichtet worden waren, unter dem Titel Epistolarum ab eruditis viris ad A. Hallerum scriptarum Vol. VI (Bern 1772-1775, $8^{\circ}$ ) und Einiger gelehrter Freunde deutsche Briefe an den Herrn von Haller (Bern 1777, 80 ${ }^{2}$ eine Auswahl herausgegeben hat. Dann wurden von dem um die Wissenschaftsgeschichte so hoch verdienten Zürcher Astronomen RudoLF Wolf (1816-1893) weitere Briefe in den Mitteilungen der Berner Naturforschenden Gesellschaft ${ }^{3}$ im Auszug ver-

${ }^{1}$ Auszugsweise mitgeteilt an der 9. Henry-E.-Sigrist-Konferenz, Luzern, 30. März 1958.

${ }^{2}$ R. Wolf, Biographien zur Kulturgeschichte der Schweiz, Zweiter Cyclus, Zürich 1859, S. $123 \mathrm{f}$.

3 R. Wolf, Mitteilungen der Berner Naturforschenden Gesellschaft 1845-1848, 1850, 1851. 
öffentlicht. Der schriftliche Verkehr Hallers mit seinem Freunde J. G. ZrmmermanN (1728-1795) ist dank BoDEMANN ${ }^{4}$ und IscheR ${ }^{5}$ verhältnismäßig am besten bekannt. Die Briefe Hallers an die Basler Botaniker wurden von Hermann Christ (1833 bis 1933) publiziert ${ }^{6}$. Die Krone hat dem ganzen editorischen Euvre der naturwissenschaftlich orientierten Briefe Hallers der damals 32 jährige SigERIST aufgesetzt, der die Briefe Hallers an Johannes Gessner (1709-1790), seinen besten Freund in der Schweiz, in einem mehr als 500 Seiten umfassenden Band herausgegeben hat. Besondere Beachtung verdient die Tatsache, daß die Briefe ungekürzt abgeschrieben sind, eine bei der schwer leserlichen kleinen Schrift Hallers um so verdienstvollere Leistung ${ }^{7}$.

Was die Leistung Hallers in bezug auf die Elementa Physiologiae gesamthaft betrifft, so ist eine auch nur summarische Würdigung ihres Inhalts hier nicht möglich. Es soll vielmehr versucht werden, vor allem anhand der genannten Briefausgaben von Sigerist und Bodemann ${ }^{\text {7a }}$ die Entstehung zeitlich zu fixieren, die Verleger des Werkes, die Arbeitsweise Hallers und die Nachwirkung innerhalb der Physiologie anhand einiger Äußer ungen kurz zu skizzieren.

\section{Die Zeit der Entstehung}

Einer der interessantesten Pharmakologen des 18. Jahrhunderts, Anton voN SтоексK (1741-1803), dessen Arbeiten Haller sehr geschätzt hat, schreibt in seinem begeisterten Nekrolog ${ }^{8}$ : «Sogar seine Vorreden sind heller, kräftiger und lehrreicher als manche andere Bücher und können darin nur mit denen Klopstocks verglichen werden.» Halten wir uns für den Anfang am besten an die «Praefatio» Hallers zu dem achten, letzten Band seines monumentalen Werkes, der übrigens im gleichen Jahr erschien wie die Gesamtausgabe der vom «Royal College of Physicians» besorgten Werke Harveys, die Haller sehnsüchtig erwartete.

Mit wohlverständlicher Erleichterung atmet Haller von der gewaltigen Arbeitslast auf. Mit ihm sind wir dankbar, daß ihm die Beendigung dieses

4 Eduard Bodemann, Von und über von Albrecht Haller. Ungedruckte Briefe und Gedichte Hallers ... Hannover 1885.

5 Rudolf Ischer, J. G. Zimmermanns Briefe an Haller, in Neues Berner Taschenbuch 1904-1910.

6 Verhandlungen der Naturforschenden Gesellschaft Basel 29 (1918) 1-59.

7 H. E. Sigerist, Albrecht Hallers Briefe an Johannes Geßner (1728-1777), in Abhandlungen der Gesellschaft der Wissenschafter zu Göttingen, Mathematisch-Physikalische Klasse, N.F. Band 11, Berlin 1923.

7a Zitiert «Sigerist-Geßner» und «Bodemann».

8 Bodemann, S. 208. 
Werkes geglückt ist. Wie oft hatte er nicht selber an dieser Tatsache gezweifelt in all den Jahren, da er zwischen Hangen und Bangen um seine Zukunft und um das Wohlergehen seiner Familie hin- und hergerissen wurde. Jetzt legt er die Feder zur Seite, um für einen kurzen Augenblick zu verschnaufen und die Bilanz des gesamten Euvre zu ziehen, so wie sie sich ihm am 1. August 1765 darbot. Manche schlaflose Nacht, sei es wegen der Arbeit oder wegen des Nachgrübelns über seine Freunde und Gegner, mag ihm in der Erinnerung aufgetaucht sein. Im Rückblick denkt er bei aller Anerkennung der schönen Jahre des Statthalteramts in Roche mit Wehmut an die einstigen reichen Möglichkeiten Göttingens: «Leicht sehe ich den Mann, der, umgeben von einer großen Bücher-Sammlung, die Möglichkeit des Nachlesens jederzeit gegenwärtig hat, der seine Zweifel bei der Leichenöffnung fortlaufend beseitigen kann, dem eine Fülle von kunstvoll präparierten Teilen des menschlichen Körpers zur Verfügung steht, der das Glück hat, von keiner andern Sorge abgelenkt zu werden, ein solcher Mann würde ein viel besseres Buch als das meinige schreiben.»

Die «Elementa» sind, wie wir früher bereits gezeigt haben, von Haller als sein eigentliches Lebenswerk betrachtet worden. Es erregt deshalb etwas Befremden, wenn die neueste Ausgabe des Großen Brockhaus die ganze biologische Seite von Hallers Tätigkeit sozusagen mit Stillschweigen übergeht und außerdem mit falschen biographischen Angaben aufwartet. Der Keim zu dieser jahrzehntelangen Beschäftigung mit der Physiologie wurde ohne Zweifel in den Wochen des Winters 1728/1729 gelegt, als der junge Doktor, wenig mehr als zwanzig Jahre alt, den erkrankten Professor Johann Rudolf Mieg (1694-1733) bei den Sektionen als Lehrer und Demonstrator vertreten durfte, ein Ereignis, an das noch der alternde Mann immer voll Stolz zurückdachte. In jenen Jahren der ersten Heimkehr aus der Fremde sind ja auch die vielen Gedichte entstanden, durch die Haller in die Geschichte der deutschen Literatur eingegangen ist.

Und nun ist es die zweite Heimkehr des gereiften und vom Sturme der Göttinger Jahre geschüttelten Professors, die ihm den Schwung für eine Forschertätigkeit gibt, wie sie wohl in der Geschichte der Medizin ihresgleichen sucht. Während seiner Gouverneurstätigkeit seien die ersten vier Bücher entstanden, berichtet Haller in seinem Rückblick, eine Angabe, die nicht ganz den Tatsachen entspricht, da der erste Band schon 1757 erschien (vgl. unten). Versuchen wir daher, die Entstehung dieses fesselnden Werkes aus den Briefen Hallers an Geßner und an seinen Schüler Johann Georg Zimmermann (1728-1795) zu verfolgen. 
Ich muß gestehen, daß das Wort ${ }^{9}$ des Genfer Naturforschers und Philosophen Charles Bonnet (1720-1793) nach Hallers Tode mir ganz aus dem Herzen gesprochen ist. Bei Bodemann (S. 176) heißt es: « Je ne desire pas moins de lire l'éloge que le Marquis de Condorcet a prononcé devant l'Académie de Paris. Quelle riche matière pour un éloge (!) historique. Mais croyez, que c'est sur tout dans les lettres qu'il faut connoitre le grand Haller.» Und der etwas nüchterne Jean Senebier (1742-1798) begründet dieses Lob etwas ausführlicher ${ }^{10}$. Wir können hier nicht auf den verschiedenen Charakter der Korrespondenz eingehen, es ist nur zu bedauern, daß so viele Briefe des Meisters an seinen ersten jugendlichen Biographen verlorengegangen sind, während das Gegenstück vollständig erhalten zu sein scheint.

Verschiedene Stellen zeigen, mit welcher Wehmut Haller an Göttingen zurückdachte. Nicht nur der Mangel an Assistenten und Materialien wiegt schwer, vielmehr sind es auch einige Freunde, deren Gegenwart ihm fehlte, allen voran Paul Gottrried Werlhof (1699-1767), mit dessen Tod für Haller «alles verloren » zu sein schien ${ }^{11}$. Wenn seine Tätigkeit an der jungen Universität ihm auch viele Feindschaften schuf, worunter Haller zeitlebens schwer litt, so war er dort - vom häuslichen Leben bis zum öffentlichen Auftreten gesehen - doch der Mittelpunkt junger Menschen gewesen, und bei der angespanntesten Arbeit ist es nicht verwunderlich, daß auch Fremden später die «Reizbarkeit der Seele» als einer seiner Hauptzüge erschien. Dies führte dann zu derart unerfreulichen Vorkommnissen wie dem Streit mit seinem Basler Prosektor J. J. Huber (1707-1778). Wenn man aber die aus Göttingen an seinen Freund, den Landvogt J. R. von Sinner (1702 bis 1782 ) in Saanen gerichteten Briefe liest ${ }^{12}$, so ist die ernstgemeinte Trauer verständlich, die in vielen Briefen über das Schicksal Göttingens während des Siebenjährigen Krieges (1756-1763) zum Ausdruck kam ${ }^{13}$.

Wie trostlos es nach Hallers Heimkehr in seine Vaterstadt (1753) in der hannoverschen Universität ausgesehen haben muß, erzählt uns ein von

9 Vgl. über ihn die Arbeit meiner Schülerin B. Moeschlin-Krieg, Zur Geschichte der Regenerationsforschung im 18. Jahrhundert, Basel 1953, S. 27 f., wo auch der Briefwechsel mit Haller erörtert ist.

10 Brief an Zimmermann: Bodemann, S. 174.

11 Bodemann, S. 76. Zimmermann wurde bekanntlich WerLhofs Nachfolger als königlicher Leibarzt, nachdem S. A. Tissoт (1728-1797) abgelehnt hatte.

12 Bodemann, S. 95-121.

13 Sigerist-Geßner, S. 269; Bodemann, S. 56. Göttingen war fünf Jahre lang von den Franzosen besetzt, zeitweise hausten sechs Bataillone dort (Brief vom 18. August 1758 an Zimmermann). 
Wolf zitierter Brief an Haller aus dem Jahre 1757: «Depuis votre départ, Monsieur, de Göttingen cette académie est tombée toujours plus en décadence d'une manière remarquable.» Ein Student, der erfuhr, daß Haller nicht mehr dort sei, ließ unverzüglich sein Gepäck wieder aufladen und zog nach Leipzig weiter.

\section{Bern (Sommer 1753-1758)}

Die Rastlosigkeit der Arbeit, die ihn niemals außer seinen geliebten Klassikern Cicero, Ovid, Virgil und Tacitus irgendwie einer Muse huldigen ließ, erfüllte Haller auch in Bern. Auch hier wie vorher in Göttingen ${ }^{14}$ teilte er seine Arbeit zwischen der anatomisch-physiologischen Forschung (vor allem über den Blutkreislauf) und dem Exzerpieren von Literatur. Er berichtet selber über seine damaligen Werke, die aus Auszügen aus seinen jahrzehntelang geführten Aufzeichnungen entstanden ${ }^{15}$. Immer wieder fühlte er sich von der Praxis angezogen, die ihm auch jetzt nicht ganz fehlte.

Die Hallers ganzes Leben beherrschende Ambivalenz zwischen der Hingabe an seine Patienten und der experimentell-literarischen Arbeit geht aus folgender Briefstelle hervor: «Les livres ne menent à rien. Ne conclués rien contre moi, j'ai été araché à la pratique, que j'aurais préférée à toute autre occupation. ${ }^{16}$

Diese Stelle gibt auch Hinweise auf Hallers Gebrauch des Französischen, dessen er sich gegenüber Zimmermann oft in recht hastiger Weise bedient, während die Briefe an Geßner in gepflegtem, weithin klassisch anmutendem Latein geschrieben sind. Selbst ein Altphilologe vermöchte wohl nicht allzuviele Verstöße gegen Stil und Grammatik zu finden (einmal etwa "venibit», Sigerist-Geßner, S. 272, 13. August 1757), und wo ein Mißgeschick vorkommt, geht es auf das Konto der Hetze seines Tagewerks gerade in den Jahren des Erscheinens der «Elementa». Auch bei seinen Kindern achtete Haller auf den Stil ihrer Briefe, wenn ihm auch das «Herz» wichtiger war (Bodemann, S. 124, Brief an seine Tochter Emilie vom 14. Oktober 1764).

Bei all seiner zum Ausdruck gebrachten Affinität zur Praxis nehmen aber doch wohl die Bücher den ersten Platz ein. Er empfiehlt seinem jungen Freunde größte Sorgfalt im Umgang mit Entliehenem, denn: «Je suis pesant, j'ai la vue basse, il me faut des échafauts pour chercher les livres et je ne suis assisté de personne ${ }^{17}$. Welche intimen Einblicke in Hallers Studierzimmer gestattet etwa auch die Stelle aus einem an Geßner gerichteten Brief (17. April 1753): «Exstant utique folium Physiologiae

14 Vgl. den Brief an Zimmermann vom 7. November 1754. (Bodemann, S. 27).

15 An Zimmermann, 9. Oktober 1754 (Bodemann, S. 22).

16 An Zimmermann, 6. August 1756 (l. c., S. 48).

17 10. Mai 1755, Bodemann, S. 38. 
majoris sed nescio ubi habitat in tanta rerum mearum confusione. Is porro mihi maximus labor erit, quamprimum me exsolvero fasciculis iconum.» Dieser Anblick hätte sich uns geboten bei einem Besuch in Hallers Wohnung unmittelbar nach der Heimkehr aus Göttingen.

$\mathrm{Zu}$ diesen wohl verständlichen Schwierigkeiten kamen Spannungen zeitweise schwerer Natur mit seiner ältesten Tochter Marianne und deren Mann Fr. Ludwig Jenner, die sämtliche Briefe jener Zeit beherrschen. Auch an Intrigen aller Art fehlte es dem neugewählten Rathausammann nicht, den viele seiner Mitbürger um das offenbar begehrte Amt beneideten. Ihre praktische Arbeitskraft ist in Hallers Augen das größte Verdienst seiner Landsleute, die im übrigen weder Zeitungen noch Bücher läsen und sich von den politischen Wahlen völlig beherrschen ließen (Briefe an Zimmermann aus dem Jahre 1754). Am Ende dieses Jahres scheint sich Haller unter dem Eindruck der Bitten des Kleinen Rates endgültig entschlossen zu haben, Gesundheit, Alter und Familie zuliebe den «königlichen Bedingungen » aus Göttingen zu widerstehen und in Bern zu bleiben (SigeristGeßner, S. $227 \mathrm{ff}$.). Von jetzt an widmete er sich mit noch mehr Teilnahme auch den höheren politischen Geschäften, wodurch die Arbeit an der Physiologie immer wieder unterbrochen werde (17. Mai 1755). In kulturellen Fragen ist er ein wichtiger Experte der Regierung, und was er erreicht, macht ihm sichtlich Freude (Seminar 1755, auf Grund einer speziellen Schrift; Waisenhaus, Schulen, 1758, Landesökonomie-Kammer, Senat der Akademie; «Landsfriedliche Commissiony », 1757), auch die Kirche machte sich seine in Göttingen gesammelten Erfahrungen zunutze (Wahl ins Oberkonsistorium). Zu seinem Umzug («nunc est mutanda domus», 13. August 1757 an Geßner) kamen die Niederkunft seiner Gattin und seiner Tochter innerhalb etwa eines Monats (Juni-Juli 1758) hinzu, was indessen den Embryologen nicht von seinen Versuchen abhielt.

In die erste Zeit seines zweiten Berner Aufenthalts fällt der schwere Unfall im Rathaus (Winter 1753) ${ }^{18}$, von dem er lange benachteiligt blieb (Luxation offenbar des Ellbogens ohne Fraktur). Wie bereits von einzelnen Adressaten seiner Briefe bemerkt, klagt Haller häufig über seine gesundheitlichen Störungen, namentlich das Podagra plagte ihn später in zunehmendem Maße. Vorerst wirkte sich aber die Heimkehr recht eigentlich wundertätig aus. «Ma santé s'est assez retablie (!) et j'ai repris cette séré-

18 Vgl. dazu die immer noch beste, literaturgeschichtlich orientierte Biographie von Ludwig Hirzel, Albrecht Hallers Gedichte, Einleitung, Frauenfeld 1882, S. CCCLXV. 
nité, qui vaut mieux que le plaisir.» Er genoß die wiedererlangte Freiheit und Muße, vor allem auch die offenbar geistig anspruchslose Arbeit im Rathaus (an Zimmermann, 20. September 1754).

Vom Bildnis des Basler Malers Emanuel J. Handmann (1718-1781, porträtierte auch Leonhard Euler) im ersten Band der «Elementa » ist merkwürdigerweise in der Korrespondenz nirgends die Rede. Es zeigt Haller in der Vollkraft seiner Jahre und dürfte nach der ruhigen Heiterkeit der Gesichtszüge weitgehend der eben geschilderten Stimmung entsprechen, also in jenen Jahren entstanden sein. Die vom Verleger offenbar angekündigte «Skulptur» von JohanN Georg Wille (1715-1808), einem der bedeutendsten Kupferstecher des Jahrhunderts, auf die sich Haller besonders gefreut hatte (an Geßner, 25. August 1756), scheint also nicht zustandegekommen zu sein. Doch ist Sigerists Anmerkung (S. 255) im genannten Sinne richtigzustellen.

Roche (1758-1764)

Auch hier sei auf Grund der beiden Briefeditionen im Zusammenhang mit den «Elementa» einiges Interessante beigefügt, das uns manche Einzelheit des großen Werkes verständlich macht. Denn die «Elementa» sind zum allergrößten Teil in den Jahren von Hallers Amtstätigkeit als Salzdirektor im Schloß von Roche (Waadt) entstanden, also fernab einer wissenschaftlichen Anstalt. Der erste Kontakt mit den Salzgruben von Bex fällt, wie an Geßner berichtet wird, ins Frühjahr 1754 (l.c., S. 217); diese Inspektion wie viele andere führte Haller auf Geheiß des Großen Rates durch, sie mag ihm das Verlockende des westlichen Klimas aufgezeigt haben, denn von jener Reise an wird immer wieder der Wunsch nach einer der Präfekturen im Süden (Aigle, Nyon, Yverdon, Romainmôtier, Morges) laut. Das kältere Schenkenbergertal ${ }^{19}$ und Lenzburg wären höchstens wegen der Nähe Zürichs willkommen, wo Geßner wohnte. Im Sommer desselben Jahres wird wiederum Roche besucht, und drei Jahre später machen Versuche über die Verdunstung von Salzlösungen das Ziel «Rupe» (latin. Roche) recht eigentlich verlockend ${ }^{20}$.

«C'est le manque de subsistance qui m'a fait prendre Roche», schreibt Haller später an seinen Kollegen Zimmermann in Brugg (21. September 1761) und an seine Tochter Emilie. So wurden nach der Wahl im Frühjahr die Bücher verpackt. Von den sechs Amtsjahren erhoffte sich Haller «Freiheit und Muße» vor allem für sein Lieblingskind, die «Elementa». In den

19 Nähe Bad Schinznach, einem Teil der Aargauer Truppen aus vielen Monaten Aktivdienst (1939/1940) wohl vertraut. Vgl. Briefe an Geßner vom 14. Februar und 12. November 1754 .

20 Ed. Sigerist, S. 274. 
späteren Briefen ist spärlich die Rede von seinen mannigfachen Unternehmungen, einer eigentlich bahnbrechenden sozialen und volkswirtschaftlichen Betätigung, die man in eigens verfaßten Schriften kennenlernt. Um so mehr bieten die Briefe an Geßner wertvolle Einblicke in die botanischen Exkursionen mit ihrem oft reichen Ertrag und in einzelne administrative Geschäfte, die durch die späteren stellvertretenden Funktionen als Statthalter von Aigle noch vermehrt wurden. In der Geschichte der Embryologie sind die Brütversuche an Hühnereiern teilweise klassisch geworden, wenn sie auch von falschen theoretischen Voraussetzungen ausgingen. Von Besuchen scheint Haller, auch abgesehen von Voltaire, der sozusagen sein Nachbar am Genfersee wurde, ziemlich verschont geblieben zu sein. Nachdem er den bedeutenden Erforscher der tierischen Regeneration, Aвraham Trembley (1700-1784), schon in Bern begrüßt hatte (Oktober 1755), machte ihm dessen Landsmann Horace Benedict de Saussure (1740 bis $1799)^{21}$ auf einer seiner unvergeßlichen Alpenreisen mehrere Besuche; mit Wärme und Herzlichkeit wurde er von dem als überlegen empfundenen großen breiten Mann empfangen, und es gingen von der gemeinsam verbrachten Woche sicher bleibende Eindrücke auf den jungen Naturforscher aus.

\section{Die Verleger}

Nachdem versucht worden ist, von den für die «Elementa» entscheidenden Lebensjahren Hallers einen knappen Begriff zu vermitteln, sei nun das Werk selber etwas näher ins Auge gefaßt. Verleger und Entstehungsweise sind bisher noch nie auch nur oberflächlich untersucht worden, und obwohl die zur Verfügung stehende Zeit keine vollauf befriedigenden Ergebnisse gezeitigt hat, sei das in Erfahrung Gebrachte kurz mitgeteilt.

An der Wahl des Bezirkes von Roche für eine Amtstätigkeit mag vielleicht die Nähe der Stadt Lausanne nicht ganz unbeteiligt sein. Dieser Stadt ließ Haller seit der erfolgreichen Reorganisation ihrer Akademie im Jahre 1757, die er zusammen mit Karl Emanuel von Bonstetten (1706-1773) unternommen hatte ${ }^{22}$, offenbar in besonderer Weise seine Förderung zuteil werden. An der Akademie der Stadt am Léman hatte sich zwanzig Jahre vorher ein aus Genf gebürtiger Verleger als Drucker einge-

21 Siehe R. Wolf, Biographien zur Kulturgeschichte der Schweiz, Vol II, S. 142 f.

22 S. F. HAAG, Neues Berner Taschenbuch 1900, S. 53-75. 
führt, der Hallers Zutrauen zu verdienen schien. Es war dies MARC-MicheL Bousquet (1696-1762), über den bisher keine vollständig befriedigende Biographie vorliegt, obschon eine solche von hohem Interesse wäre.

Antoinette Dufour hat ihre bibliothekarischen Sporen abverdient in einer Diplomarbeit der «Ecole d'Etudes Sociales» in Genf, die mir von der Direktion der Bibliothek Lausanne freundlicherweise zur Verfügung gestellt wurde ${ }^{23}$. Ohne auf die spannungsgeladene Vorgeschichte Bousquets in Genf einzugehen, sei bloß auf das Angebot des Professors Loys DE Bоснат an den unternehmenden Genfer hingewiesen, das im Jahre 1737 mit Einwilligung der hochmögenden Herren in Bern zu seiner Niederlassung in der Nähe der Kirche St-François und zu baldiger Blüte des neuen Unternehmens (Buchdruckerei, Verlag, wie vorher in Genf) führte.

Mit Haller scheint Bousquet am 9. März 1754 erstmals in Verbindung getreten zu sein, als er ihm eine unter dem Pseudonym Wolfgang Spreckius verfaßte Abhandlung über eine gedruckte verbotene Schrift vorlegte. $\mathrm{Ob}$ ihm diese Geste Hallers Gunst verschaffte, ist mir nicht bekannt. Aber jedenfalls muß der nach einem tüchtigen Drucker ausschauende Gelehrte zu dem schon berühmten Verleger vieler prominenter Männer im Ausland und in der Schweiz Zutrauen gefaßt haben. Er übergab ihm die Veröffentlichung seiner «Elementa» wie auch mancher anderer Schriften ${ }^{24}$.

Die anfängliche Begeisterung scheint jedoch bald verflogen zu sein. Wie aus den Briefen an Geßner hervorgeht, machte Bousquet schon bei den chirurgischen Disputationen gewisse Schwierigkeiten, die dann allerdings wettgemacht wurden durch die großzügige Betreuung der «Elementa». Am 25. August 1756 berichtet der ehrgeizige Autor über die großen, eleganten und gut lesbaren Typen für den Druck und die schmückenden Verzierungen, ein Versprechen, das tatsächlich gehalten wurde. Auch die große Auflage von 3000 Exemplaren beglückte Haller, enttäuschte den ungeduldigen Gelehrten aber wegen der damit verbundenen Langsamkeit des Erscheinens ${ }^{25}$. Die darauf zurückzuführenden Klagen sollten denn auch nicht so bald ver-

${ }^{23}$ Herrn Direktor G.-A. Chevallaz danke ich auch an dieser Stelle für seine Auskünfte und guten Dienste.

${ }^{24}$ Es sind dies außer den «Elementa » nach A. Dufour in chronologischer Reihenfolge, die von größerem Wert gewesen wäre als die alphabetische der Verfasserin, stichwortartig: Opuscula pathologica (1755), Parties irritables (1755), Disput. chir. (1755/56), Mouvement du sang (1756), Nature sensible (1756-1760), Formation du cœur (1758), Formation des os (1758), Disput. ad, Morb. histor. (1757 ff.).

${ }_{25}$ An Zimmermann, 24. Mai 1757: «La physiologie sort trez (!) lentement de presse, parcequ'on tire 3000 copies» (Bodemann, S. 50). 
stummen, obschon Haller über Bousquet froh sein mußte, nachdem offenbar Versuche sonstiger Unterbringung gescheitert waren (Ed. SigeristGeßner, S. 256). Auch die späteren Ärzte schulden dem Genfer Verleger Dank, denn ohne seine Großzügigkeit hätte dieses grundlegende Werk nicht die enorme Verbreitung gefunden, die seine außerordentliche Wirkung in einem weiten Umkreis erklärt. Und heute wären die Bände nicht so wohlfeil zu haben, wie beigefügt werden darf.

Worauf sich die Streitigkeiten «der Verleger» über die «Physiologia» beziehen, die Haller am 25. Juni 1756 erwähnt, ist mir nicht bekannt, aber es könnte sich um die Höhe der Auflage handeln. Sicher war schon beim ersten Band der junge Mitarbeiter Bousquets, FrançoIs Grasset (1723 bis 1789) im Spiele, der schon 1755 als Reisender in das Verlagshaus eingetreten war, sich aber bald mit Bousquet entzweite und in Lausanne einen eigenen Verlag gründete.

Die weiteren Angaben über Grasset seien dem Schreiben von Herrn Alfred Roulin, dem ehemaligen Direktor der Lausanner Bibliothek entnommen, für das ich ihm auch hier herzlich danke: «Mais cet établissement n'alla pas sans de grandes difficultés. Les intrigues de Voltaire retardèrent sa naturalisation bernoise, et les manœuvres des imprimeurs établis à Lausanne l'empêchèrent longtemps d'avoir sa propre imprimerie. Ses affaires languirent, il fut même emprisonné pour dettes, et ne rétablit sa position que grâce aux solides appuis qu'il trouva à Lausanne et à Berne, entre autres auprès du Docteur Tissot, du Grand Haller et de J. R. de Sinner. Devenu enfin Bernois et bourgeois de Vich (près de Nyon) et disposant d'un atelier d'imprimeur, il réussit à monter une nouvelle Société typographique qui devait remplacer celle de Bousquet, mort depuis quelques années. Dès lors il déploya une très grande activité, multiplia ses publications, entreprit avec l'assentiment de l'auteur une édition des œuvres complètes de Voltaire en 57 volumes, et publia coup sur coup de nombreux ouvrages du Docteur Tissot et de Haller, avec lequel il fut en correspondance pendant vingt ans (1756-1776) ... P. S. La Bibliothèque de la Ville de Berne possède une quarantaine de lettres adressées par Grasset à Albert de Haller.»

Leider war es uns wegen der knappen Zeit nicht möglich, diese Briefe einzusehen. Einen gewissen Ersatz bieten die Stellen aus dem Briefwechsel mit Geßner.

Anfangs Oktober scheint der erste Band herausgekommen zu sein. Das Porträt (vgl. oben) befriedigte nun Haller ganz und gar nicht («misera faciei meae pictura», während er vorher von «sculpuntur» gesprochen hatte ${ }^{26}$. Tatsächlich ist das Bildnis drucktechnisch ungenügend, was vielleicht zum Teil auf die Qualität des Papiers zurückzuführen ist. Auch die

26 An Geßner, 15. Oktober 1757. Hier ist von einem vierten Exemplar die Rede, das er an Geßner für Morgagni schicken will. 
Arbeit des Stechers läßt zu wünschen übrig, und es hat den Anschein, als ob der Probedruck des Porträts Haller nicht vorgelegt worden wäre.

Doch der Anlaß zu Klagen gegen Bousquet besteht weiter, indem er sich zunächst weigerte, die Schrift über die Knochenbildung und die neue Abhandlung über die Irritabilität herauszugeben. Ob Grassets Intervention schon hier den Ausschlag gegeben hat? Aus dem Zusatz könnte man schlieBen, daß Bousquet ausgesprochen merkantil eingestellt war ${ }^{27}$. Die weitere Entwicklung des Verkehrs mit Bousquet scheint jedenfalls dazu geführt zu haben, daß die späteren Nachdrucke der «Elementa», die dem Original in kurzen Abständen folgte, an die früheren Mitarbeiter von Bousquet übergegangen sind. Neben Grasset ist dies der bisher nicht näher bekannte Sigismund D'Arnay (auch Darnay), der ursprünglich bei Bousquet als Übersetzer tätig war.

Auf bibliographische Einzelheiten sei hier nicht eingegangen. Für den Freund dieser Sparte wäre es sicher reizvoll, einmal eine umfassende Zusammenstellung der Ausgaben von Hallers Schriften in Angriff zu nehmen. Statt dessen mögen noch einige spätere Bemerkungen Hallers ins Auge gefaßt werden: Auch Darnay verursachte Haller viel Ärger (s. Briefe an Geßner 1759-1762: «negligentissimus Darnay», «iners», «ingratissimus»). Und einmal droht er (gegen Bousquet?) wegen eines nicht bezahlten Honorars von Fr. 1430.- sogar mit einem Prozeß (8. Februar 1762). Von 1764 an erschienen die «Elementa» in zwei verschiedenen Verlagen (Societas typographica) in Lausanne und in Bern, so daß Haller nun von der Einhaltung der Termine besser befriedigt war.

Auch der Lausanner Verleger Grasset scheint auf seine Rechnung gekommen zu sein, da er der Physiologie einer botanischen Veröffentlichung gegenüber die Priorität gab (1. Dezember 1766).

Bei dieser Edition hat es sich um den Nachdruck der ersten Ausgabe gehandelt, bei dem Haller die Gelegenheit ergreifen will, einiges zu korrigieren und zu ergänzen. Inwiefern dies geschehen ist, wurde nicht nachgeprüft, da dies weit umfangreichere Nachforschungen erfordern würde.

Schließlich sei erwähnt, daß Haller im Spätherbst 1774 mit den Verlegern (Grasset und andern) einen Vertrag für eine nochmalige Neuausgabe abschloß, die in den Jahren 1777/1778 unter dem Titel De partium corporis humani praecipuarum fabrica et functionibus in Lausanne und Bern erschien.

27 An Geßner, 25. November 1757: «Alios puto libros habet, a quibus plus sperat.» 
Es ist zu hoffen, daß die oben von Roulin genannten Briefe Grassets an Haller weitere Aufschlüsse geben werden ${ }^{28}$.

Die obengenannte «Societas typographica» (Typographische Gesellschaft), bei der Hallers letzte Bände der physiologischen Werke teilweise erschienen sind, war «wohl das größte schweizerische Verlagsunternehmen vor 1798.» HaNs STRAHM, der Direktor der Bibliothek, fügt bei ${ }^{29}$, daß er wenige positive Angaben machen könne, da «heute noch sozusagen alle aktenmäßigen Grundlagen » fehlen. Die Auskunft seihier in extenso abgedruckt: Buchhandlung und Verlag existierten von 1758 bis etwa 1830.

«Der Begründer des Unternehmens war Vinzenz Bernhard Tscharner, einer der gebildetsten Berner seiner Zeit. Um das Jahr 1758 eröffnete er vermutlich an der Judengasse einen Bücherladen, und begann gleichzeitig mit seiner Verlegertätigkeit. Es scheint, daß im Anfang auch der italienische Flüchtling und Gelehrte Fortuné Barthélemy de Felice in der Verlegertätigkeit mitwirkte, bis er 1760 seinen Wirkungskreis nach Yverdon verlegte. Die eigentliche Gründung der Typographischen Gesellschaft erfolgte vermutlich erst 1766. Vorher führte der Verlag den Namen ,Neue Buchhandlung“. Ihre Verlagswerke gehören mit denen des Verlages von BEAT Ludwig WALTHARD zu den besten Erzeugnissen bernischer Buchdruckerkunst.

Nach Vinzenz Bernhard Tscharners Tod übernahm sein Bruder, Nrkцaus Emanuel Tscharner, die Leitung des Unternehmens, an dem das Tscharnersche Familienkapital entscheidend beteiligt war. Unter Niklaus Emanuel Tscharner wurde Emanuel FRIEDRICH Fischer Hauptaktionär.

Zu Beginn des Jahres 1780 kam es zu einer Zweiteilung, nämlich in die ,Typographische Gesellschaft ${ }^{6}$, Buchhandlung und Verlag und in die ,Neue Typographische Gesellschaft", Druckerei und Verlag und später ebenfalls auch Buchhandlung. In diesem Jahre 1780 beginnt der Verlag der, Typographischen Gesellschaft, Bern und Lausanne“ die Encyclopédie von Diderot und D'Alembert (72 Teile in 36 Bänden und 3 Bänden Kupfer) herauszugeben. Den Druck besorgte die Druckerei der, Neuen Typographischen Gesellschaft'. Im Jahre 1788 scheint die alte Typographische Gesellschaft an den Buchhändler Franz Seizer und später an Emanuel Hortin übergegangen zu sein. Die späteren Schicksale des Unternehmens sind reichlich verworren, wie überhaupt die ganze Geschichte dieser Gesellschaft mangels Quellen vorläufig noch nicht klarzulegen ist. »

Ob Johann Heinrich Pott, der ebenfalls als Herausgeber des Neudruckes der «Elementa» zeichnet, ein Associé von Grasset war, dessen Beziehungen zur «Societas Typographica » nicht klar sind, oder ob er mit dieser letzteren verbunden war, läßt sich bisher nicht feststellen.

\section{Art der Entstehung}

Aus den Briefausgaben ergeben sich auch einige Anhaltspunkte für die Entstehungsweise der «Elementa» in den verschiedenen Lebensabschnitten

28 Vgl. auch Franz Thormann, Register zur Briefsammlung von Albrecht Haller in der Berner Stadtbibliothek. Beilage zum Bericht der Berner Stadtbibliothek über die Jahre 1933-1935.

29 Für seine Nachforschungen sei Herrn Professor Strahm auch hier der beste Dank ausgesprochen. 
Hallers. Wie bereits oben erwähnt, liegen die Keime der lexikographischen Tätigkeit schon in der ersten Zeit nach der Heimkehr des frisch gebackenen Doktors. Offenbar aus Mitteilungen von Hallers Familienangehörigen notiert Zimmermann ${ }^{30}$, daß er «schon in Bern einen erstaunlichen Folianten über wenige Paragraphen von Boerhavens Physiologie gesammelt» habe; hierin scheint der später so getreulich befolgte Grundsatz befolgt worden zu sein, niemals ohne Feder in der Hand zu lesen. Vorarbeiten auf die «Elementa» waren dann seine Primae lineae physiologiae (1747) und der Methodus studii medici des Jahres 1751; in einem Brief dieses Jahres werden erstmals die Folia physiologiae, also wohl das Handbuch genannt ${ }^{31}$. Jedenfalls heißt es ein halbes Jahr später von der «magna physiologia», einer "Arbeit von einigen Jahren», daß sie langsam vorangehe, da (nur) einzelne Kapitel mit denjenigen des bestehenden Buches ähnlich seien ${ }^{32}$, und dies sei der Fall, obschon er den botanischen Garten zugunsten dieses «grave opus» verlassen habe ${ }^{33}$. Allzuoft werde er bei der Arbeit unterbrochen; in Göttingen war häufige Krankheit schuld daran, in Bern das Fehlen von Mitarbeitern. So setzte er seine Hoffnung auf die Präfektur. Große Sorge bereitete ihm, ob er «einen guten Kopisten» finde, der seine Schrift leserlich abschreibe. Dabei scheint er sich in Bern in dieser Hinsicht große Mühe gegeben zu haben ${ }^{34}$.

Nebenbei entstanden damals die Bibliotheca medicinae practicae, die Abhandlungen über den Blutkreislauf und auch von Auszügen für die Opuscula pathologica ist eine Woche später die Rede. Die Zeit bis morgens zwei Uhr habe er zwischen der «Anatomie» und den Abschriften aus «Vorlesungsheften » geteilt ${ }^{35}$. Er müsse in Büchern blättern, an die er nicht gedacht habe. Zweifel werden laut, ob er lange genug lebe, sein Werk zu beendigen (10. Mai 1755, an Zimmermann). Trotz den Einschränkungen in seinem Familienunterhalt tröste ihn die «Physiologie» über alles. Langsam reift der erste Band heran, die mühselige eigenhändige Abschrift während des Jahres 1756 ist wahrlich eine «elende Arbeit» ${ }^{36}$.

Im ersten Brief aus Roche an seinen Zürcher Freund verkündet er voll Freude, daß ein Geschenk des dänischen Königs Friedrich V. angelangt sei,

30 Bodemann, S. 213.

31 Sigerist-Geßner, S. 179 (28. August 1751).

32 Sigerist-Geßner, S. 183 (3. Februar 1752).

33 Sigerist-Geßner, S. 189.

34 An Zimmermann (Bodemann, S. 21, 2. Oktober 1754.)

35 Ibid., 7. November 1754 (Bodemann, S. 27).

36 Sigerist-Geßner, S. 256 (14. September). 
dem er die beiden ersten Bände gewidmet hatte ${ }^{37}$. Die Weiterarbeit wird damals für ein ganzes Jahr durch die "Commentaria» unterbrochen, von denen allerdings die Physiologie auch profitiere.

Es dürfte sich erübrigen, das Fortschreiten des Werkes im einzelnen zu verfolgen. Eine Zusammenstellung der Daten aus den Briefen nach Zürich mag immerhin das unglaubliche Arbeitstempo bei diesem nebenamtlichen Unternehmen illustrieren :

15. Juni 1759: $\quad$ 3. Band, 8. Buch über die Atmung beendigt (S. 298);

9. September 1760: 3. Band, 4. Teil beendigt (S. 309);

16. Dezember 1760: 4. Band, Kapitel über Gehirn beendigt;

20. März 1761: $\quad$ 4. Band beendigt, 5. Band angefangen (S. 312);

1. Mai 1761: 5 . Band bis «Tactus» beendigt (S. 314);

30. November 1761: Arbeitet am «Sehen» (S. 320);

17. Mai 1762: $\quad$ 6. Band in Bearbeitung (S. 325);

26. Juli 1762: $\quad$ Mühsames Fortschreiten bei gegenwärtiger Hitze (S. 327);

7. August bis 25. Oktober 1763: Langsame und schwierige Untersuchungen über «Generations-Organe» wegen der Experimente an graviden Tieren (S. 338 ff.);

2. November 1764: 6. Band an Geßner geschickt (S. 356);

28. März 1765: 7. Band erschienen (S. 360);

1. August 1765: «Addenda» in Bearbeitung (S. 367) «allein 100 Seiten».

Im Jahre 1764 ist das Ende auf zwei Jahre später bereits abzusehen, und über all der Fron ist es verständlich, daß Haller die unendliche Kleinarbeit gelegentlich als törichterweise in die Länge gezogen empfindet (15. September 1765).

Von besonderem Reiz ist es, zu sehen, wo sich Haller am ehesten auf Hilfe angewiesen sieht. Dies ist der Fall bei der mechanistischen Erklärung der Natur, der gegenüber er sich allerdings reserviert verhält ${ }^{38}$. Er läßt die «sciences» als einen "hochet à l'humeur inquiète de l'homme» gelten, die ebenso unschuldig seien wie «die mechanischen Künste» ${ }^{39}$. Entsprechend seiner bekannten, aus der Erziehungsmethode verständlichen Abneigung gegen den Cartesianismus erscheint ihm auch die Iatrophysik immer wieder als hypothetisch; und so konsultiert er seinen Zürcher Freund, der ein besserer Mathematiker sei, über die Bewegung der schweren Partikel des Blutes unter den leichten und möchte zur theoretischen Begrün-

37 «Duos amplissimos aureos nummos dono misit» (27. Oktober 1758).

${ }^{38}$ Sigerist-Geßner, S. 220 : Stellungnahme gegenüber de Sauvages.

${ }^{39}$ Bodemann, S. $27 \mathrm{f}$. 
dung der Pulslehre wissen, wie sich der Axenstrom zum Abbiegungswinkel bei Gefäßabzweigungen verhalte ${ }^{40}$.

Im übrigen bieten die Briefe wenig über die Themata seiner Bände, indem er die sachliche Diskussion dem Text und persönliche Auseinandersetzungen den Vorworten überließ. Immerhin ist der literarische Streit mit seinem ehemaligen Lehrer Bernhard Siegfried Albinus (1697-1770) für Haller seelisch so zermürbend, daß er seinen Freund daran teilhaben läßt. Ohne uns zum Problem als solchem zu äußern - um dieses sollte sich ein Historiker der Anatomie annehmen - seien lediglich einige Äußerungen registriert. Schon bei der Vorarbeit zum ersten Band wirft er dem Holländer präparatorische Ungenauigkeit vor ${ }^{41}$. Der Kollege, der «Gott nicht liebt und die Menschen haßt», habe gegen ihn mysteriös und höflich geschrieben ${ }^{42}$. Bevor er die Kritik des Albinus an seinem ersten Band gelesen hatte, nimmt er sich vor, seinem Lehrer-sorgfältig und pietätvoll zu antworten ${ }^{43}$, was dann im Vorwort zum 2. Band (13. November 1759) erstmals geschah. Später wirft er ihm seinen rauhen Ton vor, gesteht aber zu, in einzelnen Punkten sich vielleicht selber geirrt zu haben ${ }^{44}$. Bis in die Vorbereitungen zum letzten Band zieht sich das Zerwürfnis, und Haller fragt sich, ob die historischen oder die anatomischen Fakten richtigzustellen seien ${ }^{45}$.

Auch die bekannte Fehde mit den Wiener Ärzten Anton de Haen (1704-1776) und Gerhard van Swieten (1700 bis 1772) spiegelt sich in den Briefen nach Zürich, und deren Empfänger wird in Hallers Antworten auf die Invektiven des Papstes der österreichischen Medizin eingeweiht ${ }^{46}$, der sich bloß auf vage Krankheiten und Autoritäten, hingegen nicht auf Experimente stütze. Sein Krieg mit ihm sei noch grausamer als derjenige gegen Albinus ${ }^{47}$. Hinter dem Verbot van Swietens, keinen Arzt in Österreich zur Praxis zuzulassen, der bei Haller studiert habe, sieht dieser die Frucht der Verleumdungen de Haens ${ }^{48}$. Später scheint der Wiener Kliniker über Bonnet die Freundschaft Hallers gesucht zu haben, und dieser ist der An-

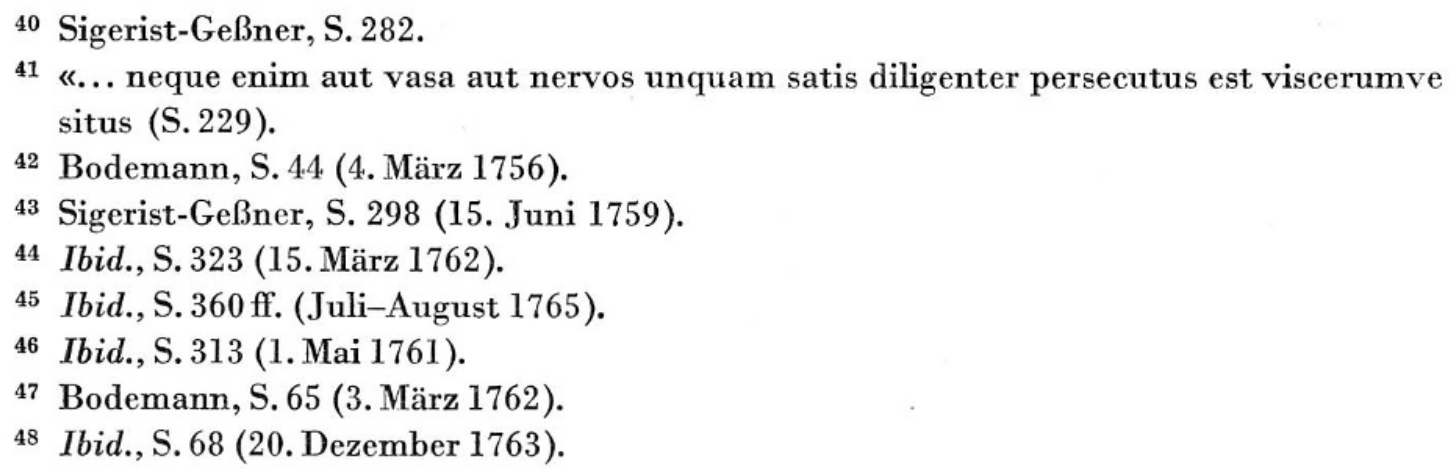


sicht, de Haen sei von allen verlassen und wolle ältere Feindschaften beendigen, um für neue Platz zu machen ${ }^{49}$.

\section{Auswirkung}

Die gewaltigen Schwierigkeiten organisatorischer Art ${ }^{50}$, die sich Haller seit seiner Rückkehr in die Heimat entgegenstellten, hielten ihn nicht davon ab, noch zwei Jahre vor seinem Tode an eine Neuausgabe seines Werkes heranzugehen. Er hoffe, darin vieles zu verbessern und zu ergänzen, die Einteilung werde vollkommener sein, und Wiederholungen seien vermieden ${ }^{51}$. Auf Wunsch des Verlegers sei der Titel abgeändert worden ${ }^{52}$. Von diesem selteneren Werk scheint eine kleinere Auflage hergestellt worden zu sein.

Zum Schluß seien noch einige Worte über die Nachwirkung der «Elementa» bei Zeitgenossen und späteren beigefügt. Durch seine Rezensionen und früher durch seine Gedichte war Haller auch in der Welt der Dichter berühmt geworden. Es ist denn auch nicht verwunderlich, daß sich JohaNn GotTfried Herder (1744-1803) seine physiologischen Belege in Hallers Werken holte, den er als den «geistvollsten Compilator des Jahrhunderts » ${ }^{53}$, bezeichnet hat. Im vierten Buch seiner großartigen Ideen zur Philosophie der Geschichte der Menschheit » ${ }^{54}$ greift er vor allem Hallers Berechnungen der Relation von Körpergewicht und Hirngewicht heraus. Hallers vergleichend-anatomischer Nachweis ${ }^{55}$, daß das relative Hirngewicht mit der Abnahme des Körpergewichts zunimmt, ist bekanntlich als «Hallersches Gesetz» in die moderne Literatur eingegangen ${ }^{56}$. Über diese spezielle Frage hinaus benützt Herder aber auch die experimentellen Befunde der Irritabilität und Sensibilität, um seine «Physiologie des Denkens» und die Lehre von der «aufsteigenden Einförmigkeit der Natur» zu begründen. Es hatte sich in dieser Berufung des Weimarer Philosophen die Prophezeiung

\footnotetext{
49 Sigerist-Geßner, S. 389 (21. Januar 1767).

50 Am 9. April 1766 schreibt H. an Zimmermann: «Trouver les livres dans ma bibliotheque est devenu pour moi un probleme (!) que je ne sais plus resoudre.» Die Bücher waren in zwei Reihen hintereinander aufgestellt (Bodemann, S. 71).

51 Sigerist-Geßner, S. 500 (September bis November 1774).

${ }^{52}$ Neuer Titel: «De partium corporis humani praecipuarum fabrica et functionibus», Bern 1777-1778. 8 Bände (Anm. H. Sigerist).

${ }^{53}$ Hirzel, Albrecht $v$. Hallers Gedichte, S. CDXXXI.

54 Ed. Theodor Matthias, Band 4, Leipzig und Wien, S. $51 \mathrm{ff}$.

55 Vgl. dazu El. physiol. Vol. 4, 1762, S. 8 (Neudruck 1766, S. 10 f.)

56 Etwa: G. von Bunge, Lehrbuch der Physiologie des Menschen, Band 1, S. 144-6.
} 
von Charles Bonnet bewahrheitet, der nach Erscheinen des letzten Bandes der «Elementa» geschrieben hatte: «Cet ouvrage immortel illustrera notre siècle ... et les philosophes le garderont à juste titre comme le plus riche trésor de vérités naturelles. ${ }^{57}$

Die ganze breite Entfaltung der Physiologie im 19. Jahrhundert ist völlig undenkbar ohne die Vorarbeiten des Berner Gelehrten. Es wäre ein reizvolles Unternehmen, gewissermaßen einen Stammbaum des Einflusses der «Elementa» im einzelnen aufzustellen. Für Deutschland verläuft die Linie ohne Zweifel über KarL Asmund Rudolphi (1771-1832), der seinen Grundriß (1821) expressis verbis auf Haller aufbaute. Nicht zuletzt des «Reichtums der Thatsachen » wegen ${ }^{58}$ ist die Parallele zum ersten Standardwerk in deutscher Sprache, dem Handbuch von Johannes Müller (1801 bis 1858, Schüler und Nachfolger Rudolphis in Berlin), besonders in die Augen springend. Diese «thematische Anregung» kommt noch bei der nächsten Generation, Du Bois-Reymond, Virghow, Friedrich MiescherHis und andern zum Ausdruck. Der Erstgenannte weist in seiner fundamentalen Gedächtnisrede auf seinen Lehrer ${ }^{59}$ auf diese Zusammenhänge hin, nicht ohne die Schwächen des Müllerschen Werkes gegenüber dem «classischen Gleichmaß der Behandlung», der «sorgfältigen Gliederung des Stoffes», der «Kunst der Übergänge» und der «Anführung der Quellen» bei Haller nachdrücklich zu betonen. Diese Eigenschaften des Schweizer Enzyklopädisten vervollständigen offenbar das, was der Elektrophysiologe als «das enge Sieb» seiner Kritik bezeichnet hatte.

Die nächste Generation der deutschen Forscher mag durch LudimaR Hermann (1838-1914) vertreten sein, der fast zwei Jahrzehnte in Zürich wirkte, und als «Enkel» sei Joh. Balthasar Lughsinger (1849-1886) ${ }^{60}$ genannt, der seine Sekretionslehre mit dem Hinweis auf Hallers umfassenden Abschnitt (Band II, Buch 7, S. 359-483) einleitet ${ }^{61}$.

Jenseits des Rheins wäre natürlich vor allem an Françors Magendie (1783-1855) zu erinnern, der in seiner Abhandlung zur Geschichte der Rückenmarksforschung (Liquor cerebrospinalis) von Hallers Werk schreibt,

57 Zitiert bei R. WoLf, Biographien zur Kulturgeschichte der Schweiz, Band 2, S. 136.

58 RudolPhi, nach WoLf, l.c.

59 Emrl Du Bors-Reymond, Gedächtnisrede auf Johannes Müller, Berlin 1860, in Abhandlungen der Berliner Akademie der Wissenschaften 1859, passim.

${ }^{60}$ Vgl. über ihn: H. R. TнüER, J. B. L., ein wenig bekannter Schweizer Physiologe, Basler Veröffentlichungen zur Geschichte der Medizin und Biologie, Fasc. II, Basel 1953.

${ }^{61}$ In L. Hermanv, Handbuch der Physiologie, Band 5, Leipzig 1880. 
es sei ihm unzählige Male passiert, daß er am Ende einer «Entdeckung» diese bereits bei Haller vorfand. Mehr als einmal habe er «dieses Unglückswerk» durchgegangen, in dem «alles zu finden sei». ${ }^{62}$

Diese paar Stellen mögen genügen, um zu zeigen, welch ungeheure Wirkung allein auf dem Gebiet der Physiologie von den «Elementa» ausging, ganz zu schweigen von der Pathologie, der der Verfasser manchen Tribut gezollt hat. Je länger man sich mit Haller befaßt, um so klarer erkennt man, $\mathrm{da} ß$ in dem Werk, dessen Entstehung hier skizziert worden ist, das letzte Geheimnis seines Ruhms liegt, eine Ansicht, in der wir durch Emanuel RADL $^{63}$ und namentlich durch Mighael Foster (1836-1907) bestärkt werden.

In seinen brillanten Lectures on the History of Physiology (Cambridge 1901) schreibt dieser feinsinnige Biologe über die Elementa Physiologiae: "The year 1757 may be regarded in a certain sense as a red letter year in the history of physiology, as marking an epoch, as indicating the dividing line between modern physiology and all that went before." In dem Jahr, da Albrecht Hallers Geburtstag sich zum 250. Male und der Todestag Johannes Müllers sich zum 100. Male jährt, war es gerechtfertigt, dieses stupende Monument schweizerischer Gelehrsamkeit in schlichter Weise in Erinnerung zu rufen.

\section{Bibliographie}

E. Bastholm, The History of Muscle Physiology, Copenhagen 1950 (Acta Histor. Scient. Natur. et medicin., Vol. VII).

Eduard Bodemann, Von und über Albrecht von Haller. Ungedruckte Briefe und Gedichte Hallers sowie ungedruckte Briefe und Notizen über denselben, Hannover 1885.

Gustav von Bunge, Lehrbuch der Physiologie des Menschen, I. Band, Leipzig 1901.

Herminie de Chavannes, Biographie de Albert de Haller, Seconde Edition, Paris 1846.

Paul Diepgen, Zwei große Naturforscher des 19. Jahrhunderts. Ein Briefwechsel zwischen Emil Du Bois-Reymond und Karl Ludwig, Leipzig 1927.

Emil Du Bors-Reymond, Gedächtnisrede auf Johannes Müller, Berlin 1860 (S.A. aus den Abhandlungen der königlichen Akademie der Wissenschaften, Berlin 1859).

Antoinetre Dufour, Le libraire-imprimeur Marc-Michel Bousquet (1696-1762). Essai bio-bibliographique. Travail présenté à l'école d'études sociales de Genève, juin 1939. Sir M(ICHAEL) Foster, Lectures on the History of Physiology During the Sixteenth, Seventeenth and Eighteenth Centuries, Cambridge 1901.

${ }^{62}$ Vgl. J. M. D. OLmsted, François Magendie, New York 1944, S. 148 f. (Journal de physiologie exp. 7, 1827).

${ }^{63}$ Geschichte der biologischen Theorien, 1. Teil, Leipzig 1905, S. 121. 
EdUARD FUeter, Geschichte der exakten Wissenschaften in der schweizerischen Aufklärung (1680-1780). Aarau / Leipzig 1941 (Veröffentlichungen der Schweizerischen Gesellschaft für Geschichte der Medizin und der Naturwissenschaften VII).

F. HAAG, Die Mission Albrecht Hallers nach Lausanne im Jahre 1757, Neues Berner Taschenbuch 1900, Bern 1899, S. 53-75.

Albrecht Haller, Bibliotheca anatomica, Tom. II, Zürich 1777.

- Elementa Physiologiae Corporis Humani, Tom. I, Lausanne 1757. Tom. VIII, Bern 1766.

- Praelectiones Boerhaavii ad proprias institutiones, Tom. I-VI, Göttingen 1739-1744.

- Sammlung kleiner Hallerischer Schrifften, II: Vorrede zum ersten Theil: Der allgemeinen Historie der Natur (Über den Nutzen der Hypothese etc.), Bern 1756.

E. Hintzsche, Tagebuch der Studienreise nach London, Paris, Straßburg und Basel 1727 bis 1728, Bern 1942 (Berner Beiträge zur Geschichte der Medizin und der Naturwissenschaften, Nr. 1.)

Ludwig Hirzed, Albrecht von Hallers Gedichte, Frauenfeld 1882 (Bibliothek älterer Schriftwerke der deutschen Schweiz, Band III).

Rudolf Ischer, Zimmermanns Briefe an Haller, 1753-1754. Nach dem Manuskript der Stadtbibliothek Bern, herausgegeben von Dr. R. I.

Neues Berner Taschenbuch auf das Jahr 1905, Bern 1904.

Balthasar Luchsinger, Physiologie der Absonderungsvorgänge, in L. Hermann; Handbuch der Physiologie, Band 5, Leipzig 1880.

J. C. MörIKofer, Die Schweizerische Literatur des 18. Jahrhunderts, Leipzig 1861.

J. M. D. Olmsted, François Magendie, New York 1944.

Henry E. Sigerist, Albrecht von Hallers Briefe an Johannes Geßner (1728-1777), Berlin 1923, Abhandlungen der Königlichen Gesellschaft der Wissenschaften Göttingen, Mathematisch-Physikalische Klasse, Neue Folge, Band XI, 2.

J. SтrонL, Albrecht von Haller 1708-1777, Gedenkschrift, Zürich 1938.

Franz Thormann, Register zur Briefsammlung von Albrecht Haller in der Berner Stadtbibliothek, Beilage zum Bericht der Berner Stadtbibliothek über die Jahre 1933-1935.

Rudolf VIRchow, Hundert Jahre allgemeiner Pathologie, Berlin 1895.

- Johannes Müller, Berlin 1858.

Rudolf Wolf, Biographien zur Kulturgeschichte der Schweiz, Zweiter Cyclus, Zürich 1859. 